\title{
L'approche scientifique de la sémiothique française: réflexions contemporaines
}

\author{
Patrícia Margarida Farias Coelho \\ Pontifical Université Catholique de São Paulo. Brésil. \\ patriciafariascoelho@gmail.com \\ ORCID: https://orcid.org/0000-0002-1662-1173 \\ Marcos Rogério Martins Costa \\ Université de São Paulo. Brésil. \\ marcosrmcosta15@gmail.com \\ ORCID: https://orcid.org/0000-0002-4627-9989 \\ Rodrigo Fontanari \\ Pontifical Université Catholique de São Paulo. Brésil \\ rodrigo-fontanari@hotmail.com \\ ORCID: http://orcid.org/0000-0001-5050-1637
}

DOI: http://dx.doi.org/10.12795/IROCAMM.2018.i1.03

\begin{abstract}
Résumé: Le sens est indéfinissable.dans sa totalité et sa complexité La sémiotique de l'École de Paris repose sur cette hypothèse pour construire son modèle théorique. Elle a ainsi comme objet d'étude le paraître du sens (GREIMAS; Courtes: 2008). De cette façon, elle propose comme outil théoriqueméthodologique un parcours génératif qui capture les effets de sens produits dans et par le texte sur ses articulations sémionarratives. Ce parcours est tripartie en niveau profond, narratif et discours, chacun a sa sémantique et sa syntaxe. Il va des éléments plus simples et abstracts (niveau profond) aux plus complexes et concrets (niveau discursif). Pour indiquer ses procédures théoriqueméthodologique, nous cherchons dans cette étude
\end{abstract}

une campagne publicitaire italienne de Benetton, lancée en 2011, intitulée "Unhate". Nous sélectionons cette pièce parce qu'elle nous offre une gamme de sens qui ne sont pas restreints à matérialité linguistique, mas ils convoquent le lecteur-consommateur à se positionner devant les polémiques sociaux et idéologiques. Comme résultat de cette discutions théorique et analytique, nous comprenons que, malgré les différences théoriques qui refletent sur le sens, la sémiotique est, parmi elles, une discipline pertinente et productive qui permet de capturer le verse et le reverse du texte.

Mots-clés: Sémiotique française; Sens; Publicité; Propagande. 


\section{Les considérations initiales}

Il y a plusieurs et distinctives théories qui se penchent sur le sens dans le champ des sciences humaines. Parmi elles, nous évoquons quelqu'unes des plus remarquables dans notre modèles théorique diffusées dans les académies brésiliennes. Nous soulignons, plus loin, les différentes perspectives sémiotiques des autres propositions d'investigation du sens. L'objectif de cet article est de présenter le modèle théoriqueméthodologique de la Sémiotique de l'École de Paris, l'appliquant à une campagne publicitaire de l'entreprise italienne Benetton. En effet, nous parcourrons, de la théorie à la pratique analytique, tout le cadre théorique de cette discipline citée au-dessus.

L'Analyse du Discours problématise les constructions idéologiques inscrites dans et par le texte, dans différentes perspectives théoriques, a savoir: Charaudeau e Maingueneau, (2004); Maingueneau (2004); Gregolin (2004); Orlandi (1999); Foucault (1997); Pêcheux (1995); Brandão (1986), parmi d’autres. On développe, aussi, I'Analyse Critique du Discours, à partir des contributions de Fairclough (2002), qui, dans un abordage interdisciplinaire, souligne les pratiques socioculturelles incrustées des manifestations du langage.

Autre courant théorique assez développée qui observe le texte au-delà de l'unité de la phrase est celui de la Linguistique textuel. Au Brésil, ses dédoublements sont fondés à partir des publications de Fávero et Koch (1983) et de Marcuschi (2005). Chercheurs comme Dijk (1997) et Ducrot (1987) ont aussi développé des recherches raffinées sur le sens: le premier problématisant la construction sémantique-cognitive et sa pratique social; le second discutant les bloques sémantiques d'argumentation construits par le locuteur en son interaction avec le locuteur.

La Sémiotique, semblable à I'Analyse du Discours, présente différents courants théoriques dans son champ d'investigation. Nous avons une sémiotique fondée pour l'américain Charles Sanders Pierce (1839-1914) qui a un substrat philosophique lourd caractérisé comme une science générale et abstract, selon Santaella (2005). La sémiotique de l'École de Moscou, à partir des études de Lotman (1996) et notamment Propp (1984), recherche les interrelations des sphères de la culture, de I'histoire et de la littérature.

Il y a encore les ramifications dans l'épistémologie des études sémiotiques. Depuis Saussure (2013), on revendique une place théorique pour la sémiotique: "Elle [sémiologie] nous apprendrait en quoi consistent les signes, quelles lois les régissent. Puisqu'elle n'existe pas encore, on ne peut dire ce qu'elle sera; mais elle a droit à 
l'existence, sa place est déterminée d'avance"' (Saussure, 2013, p. 47).

Roland Barthes (1915-1980) est un sémiologue, penseur du monde contemporain, intièrement dévoué à l'interprétation des signes verbaux et imagetiques. Le sémiologue n'a pas laissé aussi d'entendre la critique comme art, la mettant en égalité avec la littérature. Pour Motta (2012), l'embate avec les signes et ses astuces a finit pour faire de lui un philosophe de nature sceptique, position qu'en effet, il a revendiqué pour sa dernière phase.

Il faudrait encore distinguer sémiologie de sémiotique dans les études dites "sémiotiques". Ce dernier s'engage, dans le cas de la sémiotique française, à construire une discipline qui puisse rendre compte des effets de sens dans les textes. La sémiologie, par son tours, élucide les lois qui organisent les signes.

Il y a encore d'autres courants théoriques qui délimitent différemment le terme sémiotique, soit dans une définition plus large, soit dans une définition plus étroite. Umberto Eco, par exemple, la définit d'une façon particulière: comme un programme de recherche qu' "étudie tous les processus culturels comme des processus de communication" (Eco, 1976, p. 8). Cependant, l'italien ne s'inquiète pas en produire un modèle général pour édifier une discipline, car, en sa perspective:

La sémiotique se préoccupe avec tout ce qui peut être pris comme signe. Un signe est tout qui peut être pris comme substituant significativement autre chose. II ne faut pas que cette autre chose existe ou soit réellement en quelque part dans le moment où un signe le représente. Ainsi, la sémiotique est, en principe, la discipline qui étudie tout qui peut être utilisé avec objectif de mentir. (ECO, 1976, p. 7).

Tout au contraire de ces propositions, Algridas Julien Greimas (1917-1992), dès la publication de Sémantique structurale, en 1996, a établit les balises dont, aujourd'hui, on nomme de Sémiotique Discursive. Greimas pour décrire la production et la compréhension des discours et leurs manifestations textuelles a conçu la génération du sens comme un parcours qui va du plus simple et abstract au plus complexe et au plus concret. Nous expliquons cette perspective sémiotique. 


\section{Sémiotique de l'École de Paris: fondements théoriques}

Pour Greimas et Courtés (2008), la lecture de quelques matérialité linguistique n'est ni transparente ni peut se circonscrire à une référence empirique. Cette proposition (re)interprète la pensée du maître genevois Saussure (2013) et du linguistique Louis Hjelmslev (2009).

De Saussure la sémiotique récupère la notion de valeur: "Puisque la langue est un système dont tous les termes sont solidaires et où la valeur de l'un ne résulte que de la présence simultanée des autres" (Saussure, 2013, p. 161). Greimas et Courtés (2008) la définissent ainsi: "Toute sémiotique n'est sinon un réseau de relations". (Greimas et Courtés, 2008, p. 502).

De cette façon, par le regard sémiotique, le sens ne naît pas exactement du référent, cependant il peut se pointer vers lui. "Bien loin de dire que l'objet précède le point de vue, on dirait que le point de vue crée l'objet; en effet, nul nos dit à l'avance que l'une de ces façons de considérer le fait en question est antérieur ou supérieur à d'autre". La perspective de la sémiotique est alors immanente, car déduire le sens dedans la propre structure que l'organise.

Hjelmslev comprend pour structure "une entité autonome de dépendances internes" (Hjelmslev, 1991, p. 29). De cette façon, dans le domaine de la sémantique comprise comme l'étude du sens -, le structuralisme a pour l'objet non le signifié, mais la signification, c'est-à-dire, "les valeurs linguistique définis par les positions relatives des unités dans l'intérieur du système" (Hjelmslev, 1991, p. 38). On confirme ainsi le sens déduit de l'immanence du texte, autrement dit, de sa forme et pas strictement de sa substance.

D’après Hjelmslev, la sémiotique partage encore la thèse suivant: "tout processus correspond à un système qui permet de l'analyser et décrire à travers un numéro restreint de premisses" (Hjelmslev, 2009, p. 8). Car, la perspective sémiotique est générale et syntagmatique: elle décrit l'énoncé, l'instance présupposée, et récupère, à partir de cela, l'instance présupposant, l'énonciation, qui organise tout et quelque type de texte.

Dans cette conjecture, "introduire la notion de structure dans l'étude des faits sémantique, c'est introduire la notion de valeur côté à côté avec celle de la signification" (Hjelmslev, 1991, p. 118). Le sens est ainsi, en soi-même, indéfini; il ne devient que déductible par le prisme de la sémiotique, d'après la pensée 
hjelmslevienne, à partir des relations que les établaient. Autrement dit, il ne devient qu'une entité autonome passif d'analyse quand nous arrivons à comprendre ses dépendances internes. La sémiotique de courant française est, par conséquence, immanente, structurel, général et syntagmatique.

À partir des postulés saussuriens et hjelmesleviens, la sémiotique greimasienne propose chercher le texte verbal, visuel ou syncrétique en rapport entre le plan d'expression (le signifiant saussurien) et le plan du contenu (le signifié saussurien). De fait, selon cette proposition théorique, nous n'avons pas accès à réalité en soi-même, mais l'empreint de réalitéproduite dans et par les rapports entre les instances d'énoncé, le dit, et d'énonciation, le dire.

D'après cela, Greimas et Courtés (2008) éclairent que l'acteur sujet de l'énonciation est une entité bipartie entre actant-énonciateur et actant-énonciataire ${ }^{1}$. En fait en soimême il n'existe pas, elle est une (re)création d'interaction entre l'énonciateur, celui qui énonce, et l'énonciataire, celui qui interprète. Ces sont des pars interchangeables et coauteurs de l'énoncé produit.

La communication est comprise comme une structure narrative minimale de change de valeurs, désirable et nocifs. L'actant-énonciateur possède un faire persuasif (faire croire) qui cherche l'adhésion de l'énonciateur. Celui-ci, à son tour, possède un faire interprétatif (acte de croire) qui peut ou pas adhérer à manipulation de l'énonciateur.

Alors, le faire de l'énonciateur et le faire de l'énonciataire sont superposés dans le contrat de véridiction du sujet de l'énonciation:

[...] un croire-vrai doit être installé aux deux extrémités du canal de la communication, et c'est cet équilibre, plus ou moins stable, cette entente tacite de deux complices plus ou moins conscients que nous dénommons contrat de véridiction (ou contrat énoncif). (Greimas et Courtés, 2008, p. 530, nous soulignons).

Le bien fonctionnement de ce contrat de véridiction permet l'interaction, la diffusion et la permanence ou l'exclusion de déterminées vérités dans la communautés humaines:

La "vérité", pour être dite et assumée, doit se déplacer vers les instances de l'énonciateur et de l'énonciataire. L'énonciateur n'est plus censé produire des discours vrais, mais des discours produisant un effet de des "vérité": de ce point de vue, la production de la vérité correspond à l'exercice d'un faire cognitif particulier, d'un faire paraître vrai que l'on peut appeler, sans aucune nuance péjorative, le faire persuasif. (Greimas et Courtés, 2008, p. 531)

\footnotetext{
1 Dans la théorie sémiotique, le métaterme actant est une fonction syntactique exercée en déterminée instance de sens. Le métaterme acteur est lexème qui possède tant la fonction syntactique d'un actant, quant une fonction thématique, c'est-à-dire, il est aussi un élément réitéré de la sémantique narrative. Un acteur peut posséder, ainsi, plusieurs rôles thématiques et syntactique dans un même texte, c'est-à-dire, assumer distinguées fonctions actancielles et thématiques dans un même énoncé.
} 
Comme nous pouvons s'apercevoir, l'objet de la sémiotique française ne réside pas simplement dans le sens. Si c'était comme cela rien le différencierait d'autres disciplines qui se dévoilent ce champ, comme la sémantique formel, la philosophie, la sociologie entre d'autres. La sémiotique française, donc, s'établit sous le postulé du paraître du sens. À partir de Bertrand (2003), nous pouvons même dire que c'est une "abordage relativiste d'un sens, sinon toujours incomplète, au moins toujours dépendent dans les trames du discours" (Bertrand, 2003, p. 21).

Dans un de ses derniers textes, le fondateur de la Sémiotique Discursive souligne:

Tout paraître est imparfait: cache l'être ; c'est à partir de lui que se construisent un vouloir-être et un devoir-être, ce qui est déjà un déviation du sens. Seul le paraître, en tant peut être - ou peut-être - est à peine vivable. Ceci dit, il constitue tout de même notre condition d'homme. (Greimas, 1987, p. 9).

Assumées ces mêmes présuppositions théoriques, le maître lituanien propose pour examiner le plan du contenu un parcours génératif du sens. Ce parcours est triparti en niveau profond, narratif et discursive, chacun avec sa sémantique et syntaxe. Selon Fiorin et Discini (2013) présentent,

Au premier palier, ce sont les oppositions sémantiques sur lesquelles on construit le discours et les opérations qui se réalisent avec elles (négation et affirmation); au deuxième, on décrit les faires (transformation d'état) d'un sujet à la recherche d'un objet; cet agir est un simulacre d'action de l'homme dans le monde et de ses relations avec les autres hommes; au troisième, on réalise thématique ou figurativement les structures narratives et aussi elles sont actorialisation, temporalisées et spatialisées. Le niveau discursif sera lié par un ou plusieurs plains d'expression, produisant textes, qui sont la manifestation du discours. Les niveaux fondamental et narratif sont "universels" culturels, sont unités discursives virtuelles qui sont à disposition du sujet parlant pour être actualisées, par l'énonciation, au niveau discursif. L'énonciation est l'instance entre ces structures et les structures du discours. (Fiorin et Discini, 2013, p. 184-185)

On comprend, dans la sémiotique française, que texte est manifestation d'un discours, c'est-à-dire, d'un contenu et encore un plain d'expression. Le discours est un niveau plus complexe et concret du plain du contenu, car il est l'unité présupposant des structures semionnaritives (niveau narratif et profond). Ce sont alors les unités présupposées du discours. Chaque culture construit ses propres relation sémionarratives, et le sujet d'énonciation, au retourner et transformer ses relations, construit son énoncé. La sémiotique gremasienne, à partir de la immanence, récupère les effets de sens qui permettent comprendre ce que le texte dit, pourquoi il dit et comme il dit. En suite, nous allons appliquer les concepts théorique-méthodologiques explicités dans cette rubrique, d'une façon à démontrer le potentiel heuristique de la 
Sémiotique de l'École de Paris dans la lecture des textes.

\section{Analyse sémiotique de la campagne publicitaire Unhate, de Benetton}

Pour mettre en évidence les procédures théorique-méthodologiques de la sémiotique française, nous recherchons, dans cette rubrique une pièce publicitaire de l'entreprise italienne Benetton, parue en 2011, intitulée Unhate. Nous sélectionnons cette campagne publicitaire car elle nos offre une gamme de sens qui ne sont pas restreints à la matérialité linguistique, mas elles convoquent le lecteur-consommateur à se positionner, à faire-faire. D'après Coelho et Costa (2013), "comme toute communication, la publicité est manipulation [...] et c'est proprement cette action résultant qui stimule, à son tour, le destinateur-entreprise à réaliser une publicité" (Coelho et Costa, 2013, p. 111).

Nous présentons, ci-dessous, quelques affiches publicitaires qui composent cette polémique campagne de la Benetton, lancée au mois de novembre de 2011:

Image 1:

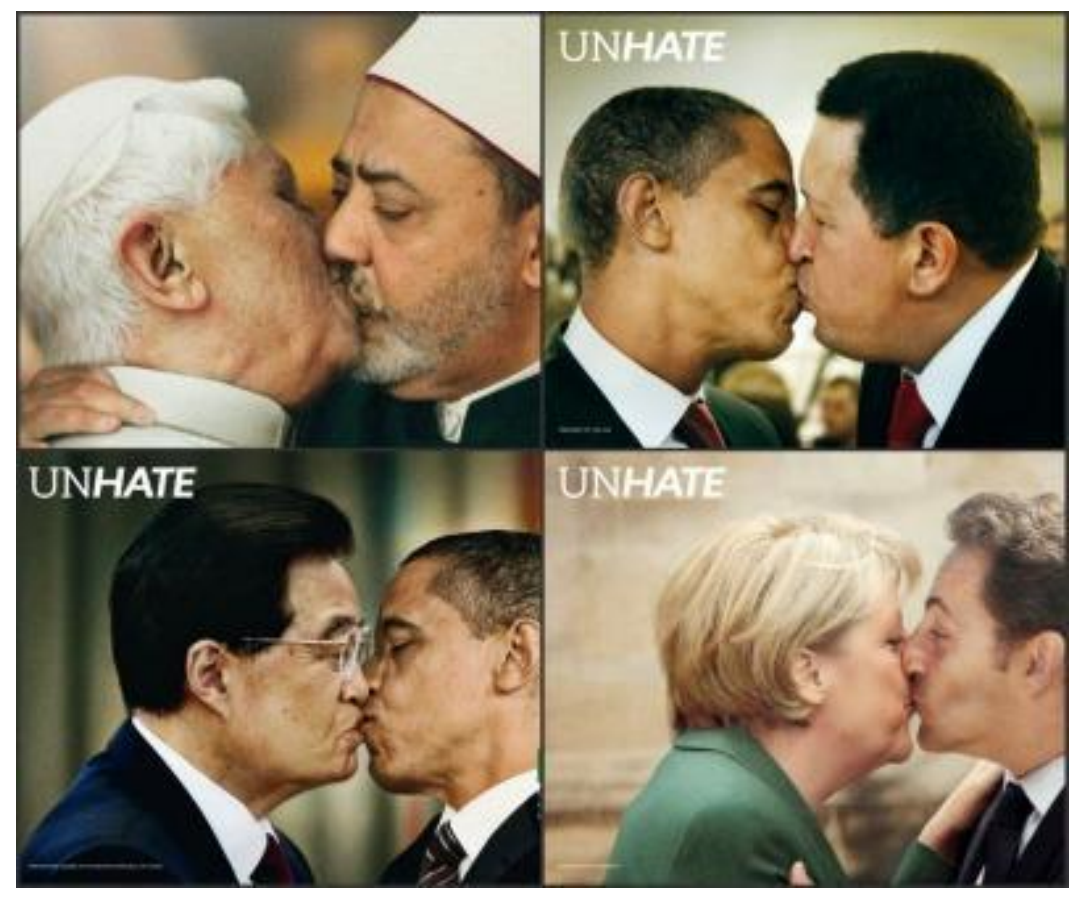

Source: Unhate Foundation - United Colors Benetton <http://unhate.benetton.com/>

Benetton est une entreprise multinationale italienne qui œuvre dans le domaine de la mode, de l'habillement: des vêtements, des accessoires et des parfums. La marque possède plus de 6.500 points de vent, distribués autour du monde en plus de 120 pays et affichant un chiffre d'affaires total de plus de 2 milliards d'euros. Ce groupe Benetton opte pour des campagnes et des slogans de conscientisation, généralement, 
sur des thèmes provocateurs comme: des races, des croyances, des ethnies. Benetton est, actuellement, une multinationale consacrée dans le marché, tant pour ses revenus, quant pour son mode recourant de construire son image devant son "public cible": une marque inscrite dans et par le polémique.

Dans la campagne publicitaire de 2011 intitulée Unhate ("non-haine"), l'entreprise expose des dirigeants du monde rivaux s'embrassant. De cette façon, le président Barack Obama (Etat unis) et Hugo Chavez (Venezuela) se baisent et la même action se répète dans toutes autres versions de cette publicité avec différents personnalités comme les pars: le papa Benoît XVI et l'iman sunnite Al-Azhar; les chefs d'état de la Corée du Nord et la Corée du Sud; les dirigeants européens Nicolas Sarkozy et Angela Merkel.

En prenant en compte les narratives de caractère provocateur instauré dans le discours publicitaire pour promouvoir la marque Benetton, nous pouvons construire un parcours génératif de sens, tel quel proposé par la sémiotique discursive de courant française.

Au premier niveau du parcours, le plus simples et abstract, suggère la signification comme une opposition sémantique minimale. Dans le cas en examen, nous avons l'énoncé verbal «Unhate» et l'énoncé visuel des dirigeants antagoniques simulant une fraternisation. Ces éléments figuratifs du niveau discursif remettent au niveau présupposé, le fondamental, l'opposition minimale: tolérance versus intolérance.

Dans l'instance de l'énoncé, on appréhende visuellement une simulation de fraternisation entre dirigeants et/ou religieux, affirmant, ainsi la tolérance. Dans l'instance de l'énonciation où se récupèrent les discours en circulation dans I'interaction énonciateur/énonciataire, on comprend que ce goût est inusité devant l'intolérance des positions idéologiques défendues pour ces personnalités. Nous avons, ainsi, l'énoncé en provocation avec l'énonciation. On affirme la tolérance dans l'énoncé en détriment de l'intolérance récupérée dans l'énonciation.

Cette opposition minimale reste évidente si nous observons le deuxième niveau, le narratif du parcours génératif du sens. Sur ce niveau, on organise la narratif à partir du point de vie d'un sujet. Le prisme de la narrative présenté dans l'affiche publicitaire est celui de Benetton. L'entreprise, comme destinateur-manipulateur, veut que le destinateur-consommateur convainque que c'est possible de promouvoir la tolérance entre sujets qui disputent le même objet-de-valeur. Le destinateur-manipulateur arrive euphoriser la tolérance, comme valeur de "Bien", en détriment de la tolérance, 
comme valeur de "Mal", sur le niveau profond.

Avec ceci, nous arrivons à récupérer le but persuasif de l'énonciateur-manipulateur de la campagne publicitaire. Sur le niveau superficiel de la lecture des annonces publicitaire, nous comprenons qu'ils promeuvent une culture contre la haine, résultant, en grande partie, de l'intolérance entre ces dirigeants et leurs positions idéologiques. Sur le niveau plus profond de la narrativité du texte, l'énonciateurmanipulateur qui mène l'énonciateur-consommateur à faire-faire, c'est-à-dire, à entrer en conjonction avec les valeurs de la marque Benetton et, par conséquence, vouloir et consommer les produits de la marque Benetton.

Sur le niveau discursif, les actants du niveau narratif s'incarnent comme marques de personnes, de temps et d'espace, construisant des figures et des thèmes ${ }^{2}$. Dans la pièce analysée, nous avons les figures des dirigeants mondiales rivaux, par exemple, Barack Oboma (EU) et Hugo Chavez (Venezuela), réalisant un geste d'apparence affectueuse: s'embrassant.

Par cette figurabilité, l'annonce publicitaire arrive à thématiser que c'est possible combattre la haine, anti destinateur de la narrative. Malgré les discours des dirigeant dans le monde naturel approuvent la manipulation de l'anti destinateur, nous pouvons faire, avec un esprit de tolérance, une culture différente de la haine. C'est l'effet de sens qui la campagne cherche éveiller dans les énonciataires des annonces.

Nous soulignons encore que la pratique réalisée par les auteurs de l'énoncé est polémique. Cela resignifie la lecture d'un baiser. Ce n'est pas quelconque baiser, c'est un baiser de sujets qui jouent, socialement, rôles biens distingués. Ils représentent des entités religieuses, des politiques nationaux et internationaux, de plusieurs instances.

En plus, tous les recours figuratifs et thématique qui composent les pars du baiser en chaque annonce sont bien distingués: un est chrétien catholique, l'autre, protestant ou islamique; un capitaliste, l'autre socialiste ; un oriental, l'autre occidental ; parmi d'autres oppositions possibles.

L'entreprise Benetton inscrit sémantiquement ses valeurs opposés dans le néologisme présenté en grosse lettres dans le centre supérieur gauche des annonces: UNHATE. Ce néologisme démontre que les mécanismes structuraux d'usage du langage sont 
affectés par des facteurs socio-historiques. La campagne a crée ce terme dans la langue anglaise associant le préfixe un au substantif hate pour que le résultant de cette composition, unhate, correspondent à une négation du sens de hate ("haine"; "haïr"; "plainte").

Ce néologisme n'est pas aléatoire. Il accompagne le système de la langue anglaise. Par exemple, nous avons unable (préfixe un + adjectif able "possible" = "impossible"), unacceptable (préfixe un + adjectif acceptable "acceptable" = "inacceptable"), unaccustomed (préfixe un + adjectif accustomed "habitué" = "déshabitué").

Nous pouvons résumer ces considérations sur le parcours génératif du sens dans cette campagne publicitaire de 2011 de Benetton dans la grille suivante:

Grille 1: Opposition entre l'être et le paraître du sens: la polémique entre les instances d'énoncée et de l'énonciation

\begin{tabular}{|c|c|c|}
\hline Title & Énonciation & Énoncé \\
\hline Niveau fondamental & Intolérance & Tolérance \\
\hline Niveau narratif & $\begin{array}{c}\text { Disjonction dos } \\
\text { actants }\end{array}$ & Conjonction des actants \\
\hline Niveau discursif & $\begin{array}{c}\text { Disputes et opposition } \\
\text { religieuse e politiques }\end{array}$ & $\begin{array}{c}\text { Fraternisation mutuelle et } \\
\text { intime }\end{array}$ \\
\hline Effet de sens & $\begin{array}{c}\text { Terme vernaculaire } \\
\text { "Hate" }\end{array}$ & Néologisme "Unhate" \\
\hline
\end{tabular}

Source: elaboration propre

Par conséquence, la position discursive assumée par le sujet de l'énonciation se met en rapport aux thèmes soutenus par les formations idéologiques de conscientisation, où la tolérance prévaut sur l'intolérance des conflits politiques et religieux, ce que confirme notre opposition minimale / tolérance versus intolérance/.

Malgré les conflits idéologiques entremêlent le plan du contenu de cette publicité depuis le niveau profond, le sujet énonciateur Benetton, au promouvoir le baiser des dirigeant antagoniques, mets l'accent sur son positionnement axiologique: l'euphorisation de la tolérance et la dysphorisation de l'intolérance.

Cette stratégie discursive de Benetton pour construire sa campagne publicitaire différencie de celle des autres marques. À la place de la multinational italienne présenter ses produits, elle utilise la publicité pour promouvoir et propager des valeurs idéologiques, dans ce cas celui d'intolérance. Cette valeur est construite discursivement par la sélection figurative et thématique du sujet de l'énonciation dans les annonces de ses campagnes. À l'absence d'un produit ou d'un objet matériel qui 
puisse remettre à un changement mercantile, se fait présent autre stratégie publicitaire: on ne montre pas les produits de Benetton, on présent les valeurs de la marque Benetton.

\section{Considérations finales}

Le sens, en sa totalité et complexité, est indéfini. La sémiotique de l'École de Paris s'appuie sous le postulé pour construire son modèle théorique. Ainsi, elle a comme objet d'étude la paraît du sens (Greimas et Courtés, 2008). Dans une certaine manière, elle propose comme outil théorique-méthodologique un parcours génératif du sens qui, comme nous avons vu, est assez efficient pour analyser les stratégies du sujet de l'énonciation.

D'après la polémique de la campagne publicitaire de Benetton de 2011, nous avons réussi à dévoiler qu'il y a des procédures discursifs et sémionarratifs qui guident et composent les effets de sens inscrits dans et par le texte. Comme la discipline général, syntagmatique, immanent et structural, la sémiotique permet d'inférer qu'au annoncer, le sujet s'énonce. C'est à partir des marques d'énonciation dans l'énoncé que nous récupérons les effets de sens que l'actant-énonciataire veut construire devant l'actant-énonciataire La pièce publicitaire d'Unhate nous offre un beau exemple de comme Benetton a organisé le rapport du sens dans ses annonces publicitaires: on privilégie la promotion d'un valeur et pas strictement celle d'un produit.

Nous ratifions, alors, que le sens n'est ni dans le référent du monde naturel, ni dans l'interprétation subjective du lecteur non plus dans le contexte social et historique qui pénètre et constitue le signe linguistique. Le sens est un paraître-être. Il est toujours inachevé, en constant construction et transformation.

Comme résultat de ces discutions théoriques et analytiques, nous comprenons que, malgré les différences théoriques qui reflètent sur le sens, la sémiotique est, parmi elles, une discipline pertinent et productive qui permet de capturer le verse et le reverse du texte. 


\section{Références}

Barros, D. L. P. (2005): Teoria semiótica do texto. 4. ed. São Paulo: ÁticaHO..

Bertrand, D. (2003): Caminhos da semiótica literária. Tradução do Grupo CASA. Bauru: EDUSC. Brandão, H. H. N. (1986): Introdução à análise do discurso. 5 ed. Campinas: Editora Unicamp. Charaudeau, P; Maingueneau, D. (2004): Dicionário de análise do discurso. Tradução de Fabina Komesu et al. São Paulo: Contexto.

Coelho, P. M. F.; Costa, M. R. M. (2013): Publicidade e contos de fadas: reflexões semióticas. Acta Semiótica et Lingvistica, João Pessoa, v. 18, n.1, p. 110-124, 2013.

Dijk, T. A. van (1997). Discourse as social Interaction. London: Sage.

Ducrot, O. (1987): O dizer e o dito. Tradução de Eduardo Guimarães. Campinas: Pontes.

Eco, U. (1976): $A$ theory of semiotics. Bloomington: Indiana University Press.

Fairclough, N. (2001): Discurso e mudança social. Tradução de Izabel Magalhães. Brasília: Editora Universidade de Brasília.

Fávero, L. L.; Kock, I. V. (1983): Linguística Textual: Introdução. São Paulo: Cortez.

Fiorin, J. L.; Discini, N. (2013): O uso linguístico: a pragmática e o discurso. In: FIORIN J. L. (Org.). Linguística? Que é isso? São Paulo: Contexto, p. 181-204.

Foucault, M. (1997): $A$ ordem do discurso. Aula inaugural no Collège de France, pronunciada em 2 de dezembro de 1970. Tradução de Laura Fraga de Almeida Sampaio. Lisboa: Relógio D'água Editores.

Gregolin, R. (2004): Discurso e mídia: a cultura do espetáculo. São Carlos: Editora Claraluz.

Greimas, A. J; Courtés, J. (2008): Dicionário de semiótica. Tradução de Alceu Dias Lima et al. São Paulo: Contexto.

Greimas A. J. (1987): De l'imperfection. Périgueux: Fanlac.

Hjelmslev, L. Prolegômenos a uma teoria da linguagem. Tradução de J. Texeira Coelho Netto. São Paulo: Perspectiva.

Greimas, A. J. (1991): Ensaios linguísticos. Tradução de Antônio de Pádua Danesi. São Paulo: Perspectiva. 
Lotman, I. (1996): La semiosfera. Tradução de Desidério Navarro. Madrid: Ediciones Cátedra.

Maingueneau, D. (2004): Análise de textos de comunicação. Tradução de Cecília P. de Souza-eSilva e Décio Rocha. 3. ed. São Paulo: Cortez.

Machado, I. (2003): Escola de semiótica: a experiência de Tártu-Moscou para o estudo da cultura. São Paulo: Ateliê Editorial.

Marcuschi, L. A. (2005): Linguística de texto. I Curso de Especialização em Leitura, Produção e Avaliação Textual (apostila). Recife.

Motta, L. T. (2012): Roland Barthes - uma biografia intelectual. São Paulo: Iluminuras; Fapesp.

Orlandi, E. (1999): Análise de discurso: princípios e procedimentos. Campinas: Pontes.

Pêcheux, M. (1995): Semântica e discurso: uma crítica à afirmação do óbvio. Tradução de Eni Pulcinelli Orlandi, Lorenço Chacon J. Filho, Manoel Luiz Gonçalves Corrêa e Silvana M. Serrani. Campinas: Unicamp.

Propp, V. I. (1984): Morfologia do conto maravilhoso. Tradução de Jasna Paravich Sarhan. Rio de Janeiro: Forense Universitária.

Santaella, L. (2005): Matrizes da linguagem e pensamento: sonora, visual e verbal. São Paulo: Iluminuras.

Saussure, F. d. (2013): Curso de linguística geral. Tradução de Antônio Chelini, José Paulo Paes e Izidoro Blikstein. 28 ed. 1 reimpressão. São Paulo: Cultrix. 